\title{
An Improved Double-threshold Cooperative Spectrum Sensing Algorithm
}

\author{
Yongxiu Feng ${ }^{1}$, Dengyin Zhang ${ }^{2}$ \\ Nanjing University of Posts and Telecommunications, Nanjing, 210003, China \\ 1email: fyxforever@126.com, ²email: zhangdy@njupt.edu.cn
}

Keywords: cooperative spectrum sensing; double-threshold; energy detection

\begin{abstract}
In order to improve the detection performance of double-threshold cooperative spectrum sensing scheme (DCSS), we provide an improved double-threshold cooperative spectrum sensing algorithm (IDCSS). Differing from DCSS, IDCSS doesn't always need all CR users send their local sensing message to FC. In IDCSS, fusion center (FC) successively merges the sensing results from each Cognitive Radio (CR) user and meanwhile makes the decision that whether the primary user (PU) is present or not depending on current-received sensing results. Once FC can determine that PU is present, it will broadcast this message to all CR users. Then those users which still wait for sending their sensing results will stop the sending behavior. Otherwise, FC receives and merges the next CR user's sensing result continuously. Simulation results show that the IDCSS can achieve better detection performance with smaller data overhead than the DCSS.
\end{abstract}

\section{Introduction}

The common fusion methods of cooperative spectrum sensing include soft and hard fusion. Soft fusion means that FC judges the presence of PU relying on merging all CR user's energy detection values. Hard fusion means that each CR user firstly get one bit hard decision information " 0 " or " 1 " by comparing their energy detection value with the threshold, then FC makes the decision relying on merging " 0 " and " 1 " under AND-rule、OR-rule or K-RANK-rule. Different weights were assigned for each CR user to conduct cooperative spectrum sensing [1]. With the number of CR users increasing, the detection performance of this method will be limited owing to the occupation of large channel resources. To decrease data overhead, ref. [2] proposed a hierarchical cooperative spectrum sensing. Since each CR user sent their sensing results to FC by the means of time division multiplexing [3], the above methods hold large time delay, which was bad for improving the performance of spectrum sensing. Considering the disadvantages of these present algorithms, this paper proposes an improved double-threshold cooperative spectrum sensing algorithm (IDCSS). The novelty of this work lies in that, FC doesn't always need to wait for merging until receiving all CR users' sensing results and then make the judgment. So the method can reduce sensing bits and decrease time delay, thus improving the detection performance of spectrum sensing.

\section{Spectrum Sensing Based on Energy Detection}

Energy detection value of the ith CR user is given by $X_{i}=\sum_{n=1}^{M}\left|x_{i}(n)\right|^{2}$, where $M$ is the number of samples, $n=1,2, \ldots, M, x_{i}(n)$ is the received signal of the $i$ th CR user $(i=1,2, \ldots, N)$. According to the central limit theorem, $X_{i}$ approximately follows normal distribution when $M$ is large enough.

$$
H_{0} \quad X_{i} \sim N\left(M \sigma_{w}^{2}, 2 M \sigma_{w}^{4}\right), H_{1} \quad X_{i} \sim N\left(M \sigma_{w}^{2}\left(1+\gamma_{i}\right), 2 M \sigma_{w}^{4}\left(1+2 \gamma_{i}\right)\right)
$$

Where $H_{0}$ and $H_{1}$ denote absence and presence of PU, respectively, $\gamma_{i}$ is SNR of the $i$ th CR user, $\sigma_{w}{ }^{2}$ is noise variance. Following the model of noise uncertainty and using unit variance we can get the two local thresholds $\lambda_{1}=\left(Q^{-1}(\alpha) \cdot \sqrt{2 M}+M\right) / \rho$ and $\lambda_{2}=\left(Q^{-1}(\alpha) \cdot \sqrt{2 M}+M\right) \rho$ at a fixed false 
alarm probability $\alpha$, where $Q^{-1}(x)$ is the inverse function of $Q(x)$ [4] and $\rho$ indicates noise uncertainty. Denoting $R_{i}$ is the ith CR user's local sensing result, we can get

$$
R_{i}= \begin{cases}X_{i} & \lambda_{1} \leq X_{i}<\lambda_{2} \\ L_{i} & \text { otherwise }\end{cases}
$$

Where $L_{i}=\left\{\begin{array}{ll}0 & X_{i}<\lambda_{1} \\ 1 & X_{i} \geq \lambda_{2}\end{array}\right.$. DCSS [5] is a hybrid method, which uses both hard and soft fusion rule. If FC makes the final decision under OR-rule, we can get the decision principle $F=\left\{\begin{array}{ll}H_{1} & S+\sum_{i=1}^{N-k} L_{i} \geq 1 \\ H_{0} & \text { otherwise }\end{array}\right.$, where $S=\left\{\begin{array}{ll}0 & X(k)<\lambda(k) \\ 1 & X(k) \geq \lambda(k)\end{array}\right.$ is soft fusion results, $X(k)=\sum_{i=1}^{k} X_{i}$ is sum of $k$ users' $X_{i}$ and $\lambda(k)=\left(Q^{-1}(\alpha) \cdot \sqrt{2 k M}+k M\right)[4]$ is corresponding threshold when performing soft fusion.

\section{The IDCSS Algorithm Description}

The CR system consists of a FC, a PU and $N$ CR users. Among $R_{i}(i=1,2, \ldots, N)$, we mark $K$ hard decision information and $N-K$ soft decision information as $\left(R_{1}, \ldots, R_{K}\right)$ and $\left(R_{K+1}, \ldots, R_{N}\right)$.

The steps of IDCSS are as follows:

1) Each CR user $i$ detects PU signal and get $R_{i}$ according to (2); 2) FC receives $R_{i}$ from CR user $i$ and determines that the PU is present directly once the results are " 1 ", then switching to 6), otherwise, $i=i+1$, and repeating 2) until $i=K-1$; 3) If $i=K$, FC cannot judge the presence of PU currently, then it will receive and merge the rest $N$-K CR users' $X_{i}$ in non-decision region; 4) When receiving the $k$ th CR user's $X_{i}$, FC calculates $X(k)=\sum_{i=K+1}^{K+k} X_{i}$ and compares $X(k)$ with $\lambda(k)$. If $X(k) \geq \lambda(k)$, FC determines that PU is present and turns to 6), otherwise, $k=k+1$ and repeating 4 ) until $k=N-K-1$; 5) If $k=N-K$, FC has received all CR users' $R_{i}$. Comparing $X(k)=\sum_{i=K+1}^{N} X_{i}$ with $\lambda(N-K)$, FC can judge the PU is present when $X(k) \geq \lambda(N-K)$, otherwise, it considers PU is absent; 6) FC broadcasts this testing information to all CR users. When all users receive the message, those users which still wait for sending their sensing information will stop the sending behavior and wait for next testing periodic time.

\section{The IDCSS Performance Analysis}

Referring to [5], we know that there exists $\gamma_{\text {min }}(k)$ to make the CR system's detection probability $P_{D}$ is not lower than the target detection probability $\beta$. When sensing channels are Rayleigh fading channels, $\gamma_{i}$ is a random variable and follows exponential distribution. If the false alarm probability and detection probability of CR user $i$ want to reach $\alpha$ and $\beta$, there exist $\gamma_{\text {min_1 }} 、 \gamma_{\text {min_2 }}$ to match $\lambda_{1} 、 \lambda_{2}$. Defining $P_{H_{0}} 、 P_{\text {fail }}$ and $P_{H_{1}}$ as the probability when $\gamma_{i}$ lower than $\gamma_{\text {min_1 }}$, between $\gamma_{\text {min_1 }}$ and $\gamma_{\text {min_2 }}$, and higher than $\gamma_{\text {min_2 }}$, then $P_{H_{0}}=1-\exp \left(-\gamma_{\text {min_1 }} / \gamma\right), P_{H_{1}}=\exp \left(-\gamma_{\text {min_2 }} / \gamma\right)$ $P_{\text {fail }}=\exp \left(-\gamma_{\text {min_1 } 1} / \gamma\right)-\exp \left(-\gamma_{\text {min_2 }} / \gamma\right)$. Denoting $P_{K}$ is the probability when FC can judge the presence of PU after hard merging $K$ CR users' hard decision information, then $P_{K}=\left(p_{0}\right)^{K-1} p_{1},(1 \leq K \leq N)$, where $p_{0}=P_{H_{0}} /\left(P_{H_{0}}+P_{H_{1}}\right)$, and $p_{1}=P_{H_{1}} /\left(P_{H_{0}}+P_{H_{1}}\right)$. Denoting $P_{N, K}$ is the probability when FC can judge the presence of PU after softly merging $K$ CR users' $X_{i}$ of non-decision region formed by $N$ CR users, then $P_{N, K}=\int_{\gamma_{\min }(K)}^{\infty} \gamma(K)^{K-1} /\left(\gamma^{K} \cdot(K-1) !\right) \exp (-\gamma(K) / \gamma) d \gamma(K),(1 \leq K \leq N)$. Assuming that the coding bits of local detection values are $w$, we calculate $P_{D}$ and the average data bits $W$. 


\section{1) $N$ CR users' $R_{i}$ fall into decision region}

Denoting $P_{K}^{\prime}$ is the probability when FC can judge the presence of PU after exactly hard merging the $K$ th user's $R_{i}$, then $P_{1}^{\prime}=P_{1}, P_{K}^{\prime}=\left(1-P_{1}\right) \cdots\left(1-P_{K-1}\right) P_{K},(2 \leqslant K \leqslant N-1)$. If FC cannot make the decision, it will use the $N$ th user's $R_{i}$, then $P_{N}^{\prime}=\left(1-P_{1}\right) \cdots\left(1-P_{N-1}\right)$. The probability of making a judgment " 1 " is $P_{d 1}=1-\prod_{K=1}^{N}\left(1-P_{K}\right)$ and the average bits $W_{1}=P_{1}^{\prime} \cdot 1+P_{2}^{\prime} \cdot 2+\ldots+P_{N-1}^{\prime} \cdot(N-1)+P_{N}^{\prime} \cdot N$.

\section{2) $N$ CR users' $R_{i}$ fall into non-decision region}

Denoting $P_{N, K}^{\prime}$ is the probability when FC judges the presence of PU after exactly softly merging the $K$ th CR user's $R_{i}$, then $P_{N, 1}^{\prime}=P_{N, 1}, P_{N, K}^{\prime}=\left(1-P_{N, 1}\right) \ldots\left(1-P_{N, K-1}\right) P_{N, K},(2 \leqslant K \leqslant N-1)$. If FC cannot make decisions, it will use the $N$ th user's $R_{i}$, then $P_{N, N}^{\prime}=\left(1-P_{N, 1}\right) \ldots\left(1-P_{N, N-1}\right)$. The probability of making a judgment " 1 " is $P_{d 2}=1-\prod_{K=1}^{N}\left(1-P_{N, K}\right)$ and the average bits $W_{2}=P_{N, 1}^{\prime} \cdot w+P_{N, 2}^{\prime} \cdot 2 w+\ldots+P_{N, N}^{\prime} \cdot N w$.

\section{3) $K$ CR users' $R_{i}$ fall into decision region and the rest $N$ - $K$ fall into non-decision region} If $K=N$, this case is equivalent to the above 1 ). If $K=0$, this case is equivalent to the above 2 ). So, the average bits and detection probability of IDCSS are

$$
\begin{aligned}
W= & C_{N}^{0} P_{f a i l}^{N} \cdot\left(P_{N, 1}^{\prime} \cdot w+P_{N, 2}^{\prime} \cdot 2 w+\ldots+P_{N, N}^{\prime} \cdot N w\right)+\sum_{K=1}^{N-1} C_{N}^{K}\left(1-P_{\text {fail }}\right)^{K} P_{\text {fail }}^{N-K}\left(( 1 - \prod _ { i = 1 } ^ { K } ( 1 - P _ { i } ) ) \cdot \left(P_{1}^{\prime} \cdot 1+\right.\right. \\
& \left.P_{2}^{\prime} \cdot 2+\ldots+P_{K-1}^{\prime} \cdot(K-1)+P_{K}^{\prime} \cdot K\right)+\left(\prod_{i=1}^{K}\left(1-P_{i}\right)\right) \cdot\left(K+P_{N-K, 1}^{\prime} \cdot w+P_{N-K, 2}^{\prime} \cdot 2 w+\ldots+P_{N-K, N-K-1}^{\prime} \cdot\right. \\
& \left.\left.(N-K-1) w+P_{N-K, N-K}^{\prime} \cdot(N-K) w\right)\right)+C_{N}^{N}\left(1-P_{\text {fail }}\right)^{N} \cdot\left(P_{1}^{\prime} \cdot 1+P_{2}^{\prime} \cdot 2+\ldots+P_{N-1}^{\prime} \cdot(N-1)+P_{N}^{\prime} \cdot N\right) \\
P_{D}= & C_{N}^{0} P_{\text {fail }}^{N}\left(1-\prod_{i=1}^{N}\left(1-P_{N, i}\right)\right)+\sum_{K=1}^{N-1} C_{N}^{K}\left(1-P_{\text {fail }}\right)^{K} P_{\text {fail }}^{N-K}\left(\left(1-\prod_{i=1}^{K}\left(1-P_{i}\right)\right)+\left(\prod_{i=1}^{K}\left(1-P_{i}\right)\right) \cdot\left(1-\prod_{i=1}^{N-K}\left(1-P_{N, i}\right)\right)\right) \\
& +C_{N}^{N}\left(1-P_{\text {fail }}\right)^{N}\left(1-\prod_{i=1}^{N}\left(1-P_{i}\right)\right)
\end{aligned}
$$

\section{Simulation Results}

Assuming $N=10, M=100, \alpha=0.01, \rho=1.5, w=8 \mathrm{bit}$, the ranges of SNR are $-10 \mathrm{~dB}$ to $10 \mathrm{~dB}$ and the sensing channels between PU and CR users are Rayleigh fading channels. We can obtain the value of $\gamma_{\min }(k)$ for different CR number $k$ and detection probability $\beta$, as shown in table 1 .

Table 1 Values of $\gamma_{\min }(k)$

\begin{tabular}{|l|c|c|c|c|c|c|c|c|c|c|}
\hline \multirow{2}{*}{$\beta$} & \multicolumn{10}{|c|}{$k$} \\
\cline { 2 - 11 } & 1 & 2 & 3 & 4 & 5 & 6 & 7 & 8 & 9 & 10 \\
\hline 0.90 & 0.598 & 0.810 & 0.973 & 1.110 & 1.231 & 1.340 & 1.440 & 1.534 & 1.621 & 1.704 \\
\hline 0.95 & 0.688 & 0.921 & 1.100 & 1.251 & 1.384 & 1.504 & 1.614 & 1.717 & 1.814 & 1.905 \\
\hline 0.99 & 0.874 & 1.147 & 1.356 & 1.532 & 1.688 & 1.828 & 1.957 & 2.077 & 2.190 & 2.297 \\
\hline
\end{tabular}

It can be seen from table 1 that the more CR users, the lower SNR of each user will be needed. For instance, when $\beta=0.90$, SNR is at least 0.598 when single user senses, however, the average SNR of each user only need to reach $0.810 / 2=0.405$ when two CR users participate in spectrum sensing. Figure 1 shows that when $\beta=0.90$, the average bits needed in IDCSS is lower than DCSS [5]. Figure 2 shows the detection probability versus SNR when using IDCSS and DCSS [5]. We can see that the IDCSS holds better detection performance. For example, when SNR is -6dB, the detection probability of DCSS is only 0.68 , but IDCSS reaches 0.94 . Figure 3 shows the detection probability of IDCSS versus SNR with different $N$. It can be seen that the detection probability will improve when $N$ increases. Figure 4 shows the detection probability of IDCSS versus SNR with different $\rho$. We can see that the detection probability will improve with the increasing SNR when $\rho$ is constant and improve with the increasing $\rho$ when SNR is constant. 


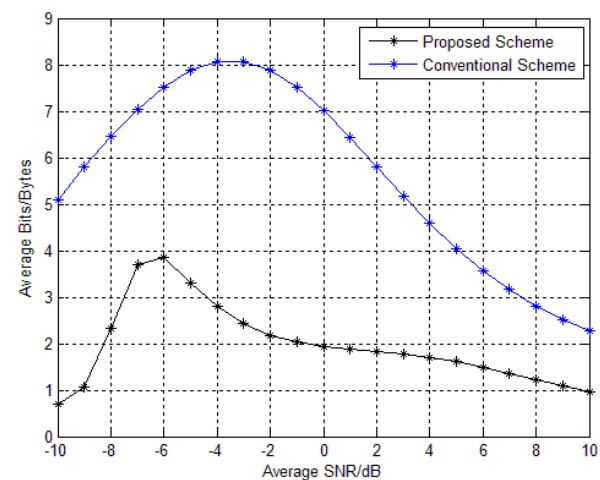

Fig.1 Average bits versus SNR

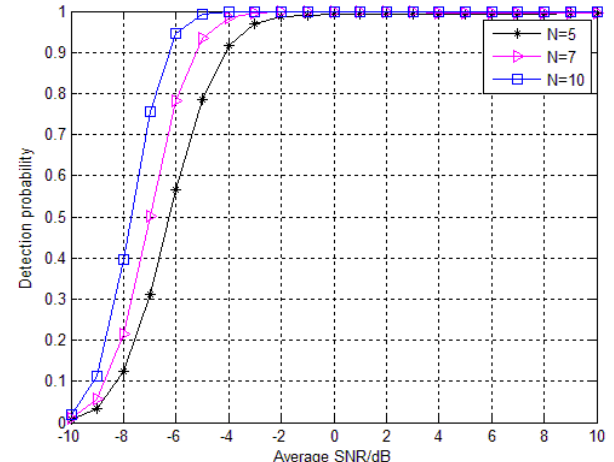

Fig.3 Detection probability versus $N$

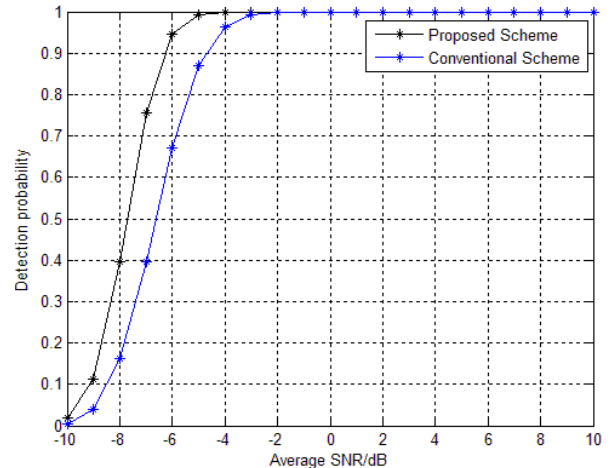

Fig.2 Detection probability versus SNR

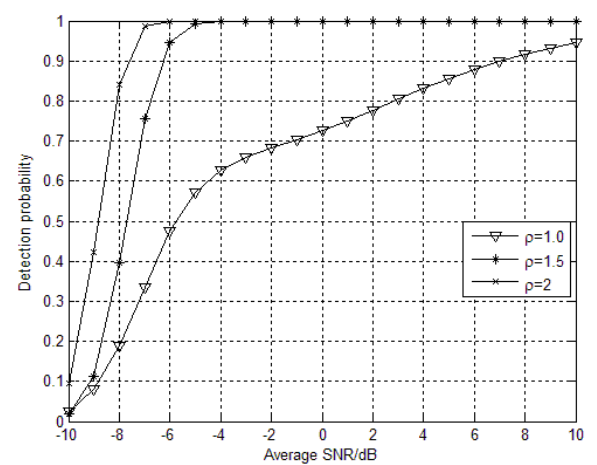

Fig.4 Detection probability versus $\rho$

\section{Conclusion}

In IDCSS, CR users in decision region send “ 0 " or " 1 ” while the rest CR users in non-decision region send local energy detection values. FC adaptively select the quantity of CR users needing to be merged according to local decision information and the change of SNR, which means that the method doesn't always need all CR users send their local sensing message to FC, so it can reduce data overhead and further improve detection performance of the CR system.

\section{Acknowledgement}

This research work is supported by the National Natural Science Foundations of P. R. China (NSFC) under Grant (61071093), National 863 Program (2010AA701202), Jiangsu Province Major Technology Support Program (BE2012849), SRF for ROCS, and SEM (NJ209002).

\section{References}

[1] S. Zheng, C. Lou, X. Yang. Cooperative spectrum sensing for cognitive radios based on a modified shuffled frog leaping algorithm [J]. Journal of Physics, 2010, 59(5):3611-3617.

[2] S. Liu, B. Hu, X. Wang. Hierarchical Cooperative Spectrum Sensing Based on Double Thresholds Energy Detection [J]. IEEE Communications Letters, 2012, 16(7):1096-1099.

[3] S. Zhang. A Low-overhead Energy Detection Based Cooperative Sensing Protocol for Cognitive Radio Systems[J]. IEEE Transactions on Wireless Communications, 2009, 8:5575-5581.

[4] Tandra R, Sahai A. SNR walls for signal detection [J]. IEEE Journal of Selected Topics in Signal Processing, 2008, 2(1): 4-17.

[5] J. Zhu, Z. Xu, F. Wang, B. Huang and B. Zhang. Double Threshold Energy Detection of Cooperative Spectrum Sensing in cognitive radio [J]. 2008. 